\title{
EDGE DETECTION BY USING LOOKUP TABLE
}

\author{
M. Sushma Sri ${ }^{1}$, M. Narayana ${ }^{2}$ \\ ${ }^{1}$ M.Tech Student, ${ }^{2}$ Professor \& H.O.D, ECE, J.P.N.C.E, Andhra Pradesh, India, \\ hiremath.sushmasri@gmail.com,sai_15surya@yahoo.co.in
}

\begin{abstract}
Edge detection is a fundamental tool used in most image processing applications. We proposed a simple, fast and efficient technique to detect the edge for the identifying, locating sharp discontinuities in an image and boundary of an image. In this paper, we found that proposed method called LookUp Table performs well, which requires least computational time as compared to conventional Edge Detection techniques. And also in this paper we presented a comparative performance of various conventional Edge Detection Techniques.
\end{abstract}

Keywords: Edge detectors, Lookup table.

\section{INTRODUCTION}

Edge Detection refers to the process of identifying and locating sharp discontinuities in an image. The discontinuities are abrupt changes in pixel intensity which characterize boundaries of objects in a scene. Edge Detection is one of the most frequently used techniques in digital image processing [10]. The boundaries of object surfaces in a scene often called lead to oriented localized changes in intensity of an image called edges [12]. Edge detection is one of the most commonly used operations in image analysis, and there are probably more algorithms in the literature for enhancing and detecting edges than any other single subject. The reason for this is that edges from the outline of an image. An edge is the boundary between an object and the background, and indicates the boundary between overlapping objects. This means that if the edges in an image can be accurately, all of the objects can be located and basic properties. Edge detection is a fundamental of low-level image processing and good edges are necessary for higher-level processing [9]. Classical methods of edge detection involve convolving the image with an operator (a 2D filter), which is constructed to be sensitive to large gradients in the image while returning values of zero in uniform regions. Edge detection technique also transforms image benefiting from changes in gray tones in the image. Edges are signs of lack of continuity and ending as a result of this formation the edge is obtained without encountering any changes in physical qualities of an image [7,17]. There are extremely large numbers of edge detection operators available each designed to be sensitive to certain edges [10]. Edge detection is difficult to implement in noisy images, since both noise and edges contains high frequency content [10].

\subsection{Edge Detector Background}

Edge detection is a tool used in most image processing application. Edge detection is an important technique in applications like object recognition, motion analysis, and pattern recognition [12]. The boundaries of object surfaces in a scene often lead to oriented localized changes in intensity of an image called edges [15.]There are many ways to perform edge detection however, majority of the different method can be grouped into two major categories:-

(a) Gradient: - the gradient method detects the edges by looking for the maximum and minimum in the first derivative of the image.

(b) Laplacian: - the Laplacian method searches for zero crossing in the second derivative of the image to find edges [7].

\subsection{Edge Detection Techniques}

There is different edge detection techniques available, the compared ones are as follows:

\section{(a) Sobel Operator}

Sobel operator is one if the pixel based edge detection algorithm. It can detect edge by calculating partial derivatives in $3 \times 3$ neighbourhoods. The reason for using Sobel operator is that it is insensitive to noise and it has relatively small mask in images. The convolution kernel, one kernel is simply the other rotated by 90degrees. These kernels are designed to respond to edges running vertically and horizontally relative to the pixel grid, one kernel for each of the two perpendicular orientations. The kernels can be applied separately to input image to produce separate measurement of gradient component in each orientation which can be combined to find the absolute magnitude of gradient at each point.

\section{(b) Robert Cross operator}

The Robert Cross operator performs a simple and quick 2-D spatial gradient measurement on an image. The operator 
consists of a pair of $2 \times 2$ convolution kernel. These kernels are designed to respond maximally to edges running at 45 degrees to the pixel grid one kernel for each of the two perpendicular orientations. The kernels can be applied separately to the input image to produce separate measurement of the gradient component in each orientation these can then be combined together to find the absolute magnitude of the gradient at each point.

\section{(c) Prewitt Detection}

The Prewitt Operator is similar to the Sobel operator and it is used for detecting vertical and horizontal edges in images [6]. The Prewitt edge detector is an appropriate way to estimate the magnitude and orientation of an edge. The Prewitt operator is limited to eight possible orientations [14] although most direct orientation estimates are not exactly accurate. The Prewitt operator is estimated in the $3 \times 3$ neighbourhood for eight directions [15]. The entire eight masks are calculated then the one with the largest module is selected.

\section{(d) Canny Operator}

Among the edge detection already discussed, the [2] Canny edge detector is the most rigorously defined operator and is widely used. It smoothes the image with appropriate Gaussian filter to reduce desired image. And it also determines Gradient magnitude and direction at each pixel. The Canny edge detector is widely considered to be the standard edge detection method in the industry. It is important that edges occurring in images should not be missed and that there be no responses to non-edges. The second criterion is that the edge points be well localized. In other words, the distance between the edge pixels as found by the detector and the actual edge is to be at a minimum. A third criterion is to have only one response to a single edge. This was implemented because the first two were not substantial enough to completely eliminate the possibility of multiple responses to an edge. Based on these criteria, the canny edge detector first smoothes the image to eliminate and noise. It then finds the image gradient to highlight regions with high spatial derivatives. Canny saw the edge detection problem as a signal processing optimization problem, so he developed an objective function to be optimized [12].

\section{IMAGE SEGMENTATION}

The first step in image analysis is segment the image. Segmentation subdivides an image into its constituent parts or objects. The level to which this subdivision is carried depends on the problem being viewed. Some time need to segment the object from the background to read the image correctly and identify the content of the image for this reason there are two techniques of segmentation, discontinuity detection technique and Similarity detection technique $[15,17]$. In the first technique, one approach is to partition an image based on abrupt changes in gray-level image. The second technique is based on the threshold and region growing. This paper discusses the first techniques using LookUp Table Edge Detection method.

\subsection{Edge Detection for Image Segmentation}

Edge detection techniques transform images to edge images benefiting from the changes of grey tones in the images. Edges are the sign of lack of continuity, and ending. As a result of this transformation, edge image is obtained without encountering any changes in physical qualities of the main image [16]. Objects consist of numerous parts of different colour levels. In an image with different grey levels, despite an obvious change in the grey levels of the object, the shape of the image can be distinguished in Figure: 6. An Edge in an image is a significant local change in the image intensity, usually associated with a discontinuity in either the image intensity or the first derivative of the image intensity. An edge is the boundary between an object and the background. The edge representation of an image significantly reduces the quantity of data to be processed, yet it retains essential information regarding the shapes of objects in the scene.

\section{PROPOSED METHOD}

The proposed method presents determining edges of images by using Lookup table and it is necessary to point out the true edges to get the best results. In this respect we present the advantages of Lookup table by comparing with conventional edge detector techniques i.e., Sobel, Robert, Prewitt, and Canny in which the LUT technique gives better detection in less time.

\subsection{LOOKUP TABLE}

In data analysis such as image processing a lookup table (LUT) is used to transform the input data into a more desirable output format. Lookup table is an array that replaces runtime computation with a simpler array indexing operation. Lookup table are often called LUT's and given an output value for each range.

\subsubsection{Applylut}

The use of applylut is it will process binary image by using lookup table. It performs 2 by 2 or 3 by 3 neighbourhood on binary image by using lookup table.

\section{(a) 2-by-2 Neighbourhood}

For 2-by-2 neighbourhoods, length (LUT) is 16. There are four pixels in each neighbourhood, and two possible states for each pixel, so the total number of permutations is $24=16$. To produce the matrix of indices, applylut convolves the binary image with this matrix.

$8 \quad 2$

$4 \quad 1$ 
The resulting convolution contains integer values in the range $[0,15]$. Applylut uses the central part of the convolution, of the same size as binary image, and adds 1 to each value to shift the range to [1,16]. It then constructs A by replacing the values in the cells of the index matrix with the values in the lut that indices the point to.

\section{(b) 3-by-3 Neighbourhood}

For 3-by-3 neighbourhoods, length (LUT) is 512. There are nine pixels in each neighbourhood, and two possible states for each pixel, so the total number of permutations is $29=512$. To produce the matrix of indices, applylut convolves the binary image BW with this matrix.

$$
\begin{array}{lll}
256 & 32 & 4 \\
128 & 16 & 2 \\
64 & 8 & 1
\end{array}
$$

The resulting convolution contains integer values in the range [0,511]. Applylut uses the central part of the convolution, of the same size as binary image, and adds 1 to each value to shift the range to $[15,12]$. It then constructs $A$ by replacing the values in the cells of the index matrix with the values in LUT that the indices point to.

\section{RESULTS \&DISCUSSIONS}

In this paper, original image is taken and the image is converted into binary image which is shown in figure (1) \& figure (2) respectively. Next for this binary image Edge techniques are implemented by using proposed method Lookup table and conventional edge techniques are Sobel operator, Robert operator, Prewitt operator, \& Canny operator and their images are shown in figure (4), figure (5), figure (6),figure (7) respectively. And the LUT image is shown in figure (3).

We found that proposed method which gives simple, fast and efficient results in less computational time. Hence the proposed method performs well as compared to conventional methods as shown in figures.

Table1: Comparison of Computational Time

\begin{tabular}{|l|l|l|l|l|l|}
\hline IMAGE & $\begin{array}{l}\text { SOBEL } \\
(\mathrm{sec})\end{array}$ & $\begin{array}{l}\text { ROBERT } \\
(\mathrm{sec})\end{array}$ & $\begin{array}{l}\text { PREWITT } \\
(\mathrm{sec})\end{array}$ & $\begin{array}{l}\text { CANNY } \\
(\mathrm{sec})\end{array}$ & $\begin{array}{l}\text { LUT } \\
(\mathrm{sec})\end{array}$ \\
\hline 1. & 0.2991 & 0.2350 & 0.1964 & 0.3566 & $\begin{array}{l}0.00 \\
84\end{array}$ \\
\hline 2. & 0.1601 & 0.0475 & 0.0257 & 0.2080 & $\begin{array}{l}0.00 \\
55\end{array}$ \\
\hline
\end{tabular}

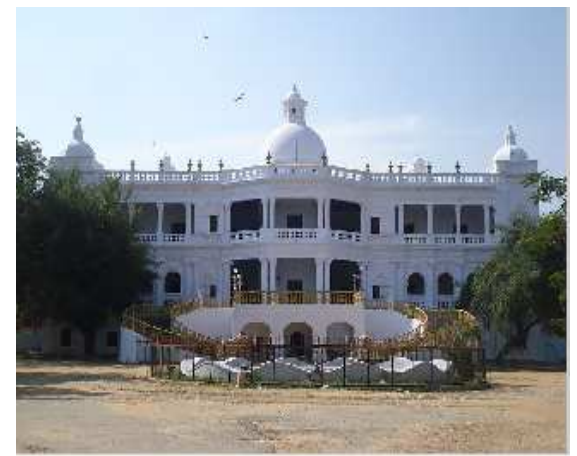

Fig1: Original Image

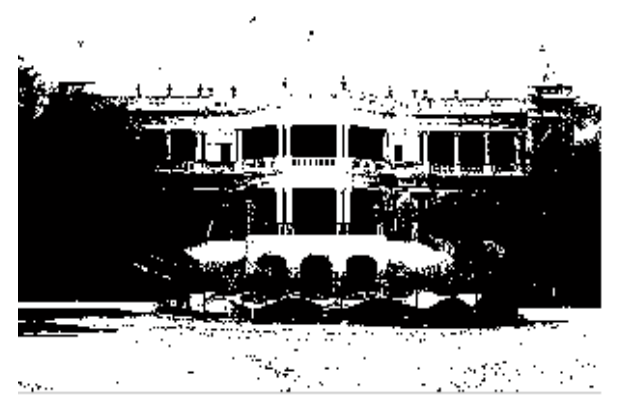

Fig2: Binary Image

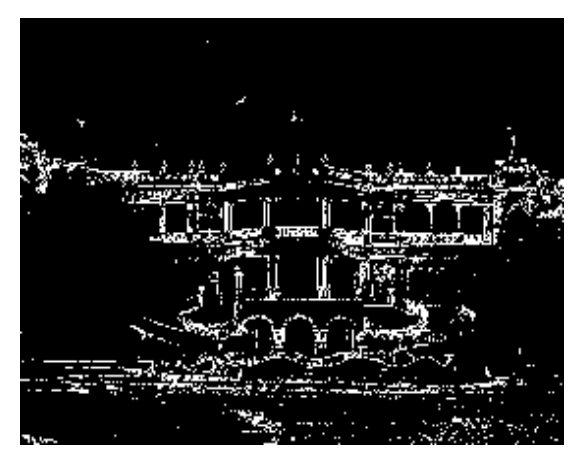

Fig3: LUT

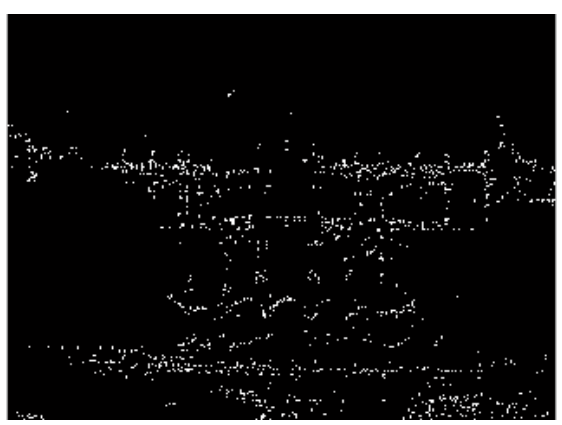

Fig4: Sobel 


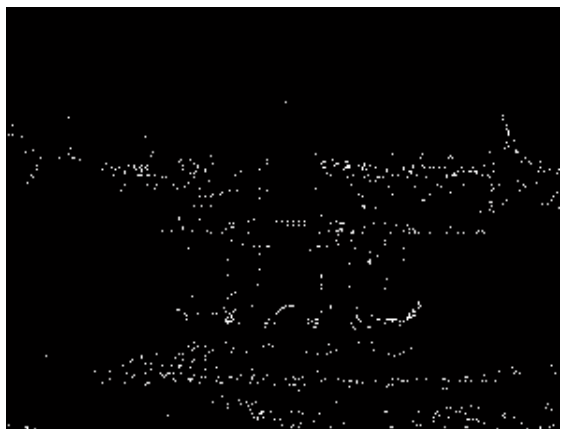

Fig5: Robert

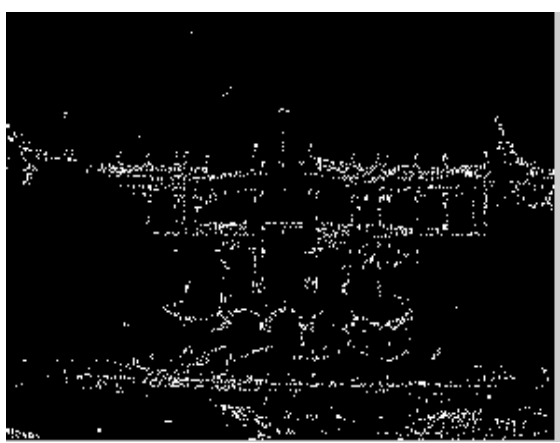

Fig6: Prewitt

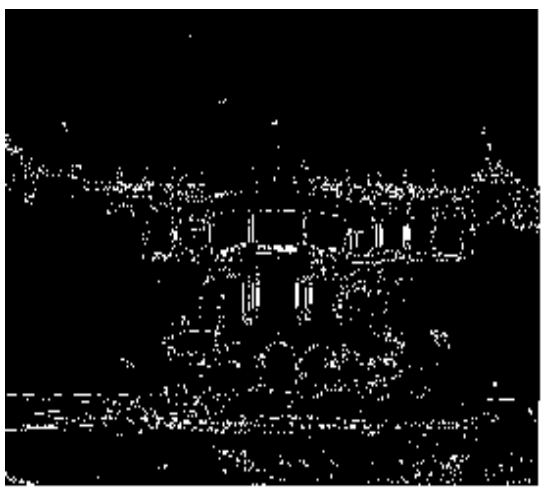

Fig7: Canny

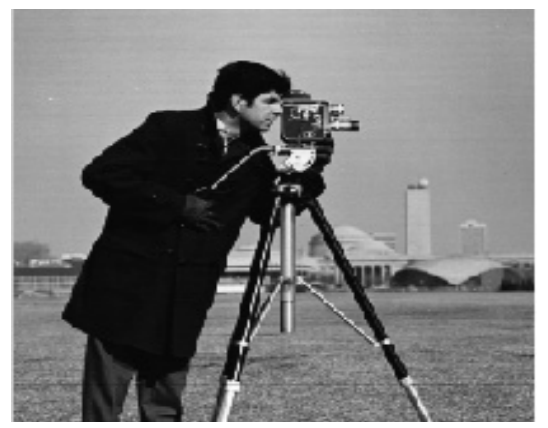

Fig8: Original Image

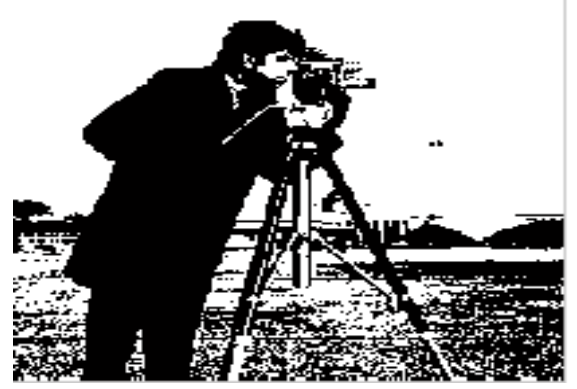

Fig9: Binary Image

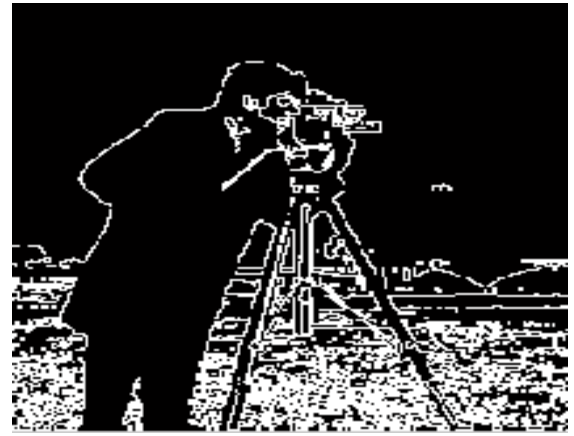

Fig10: LUT

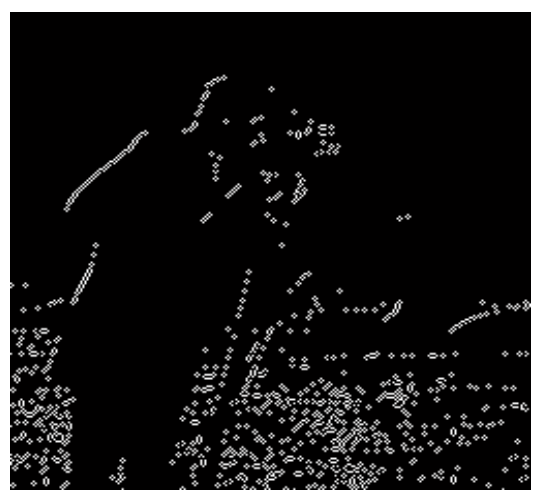

Fig11: Sobel

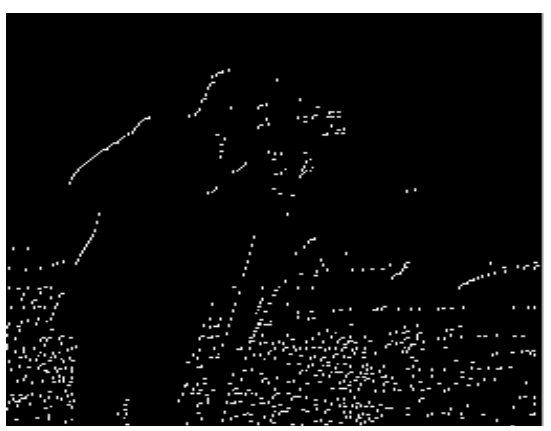

Fig12: Robert 


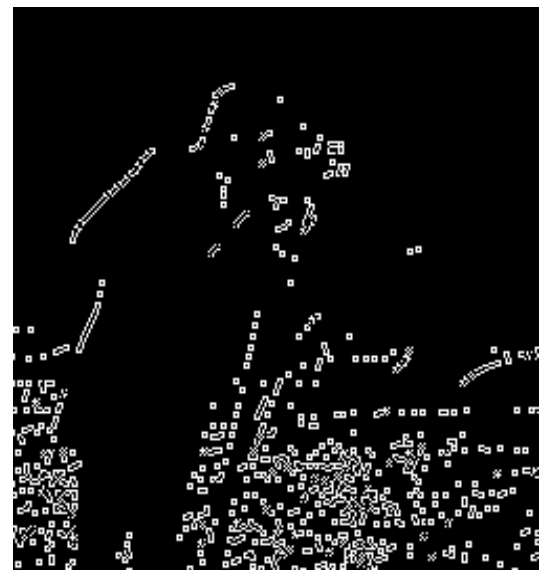

Fig13: Prewitt

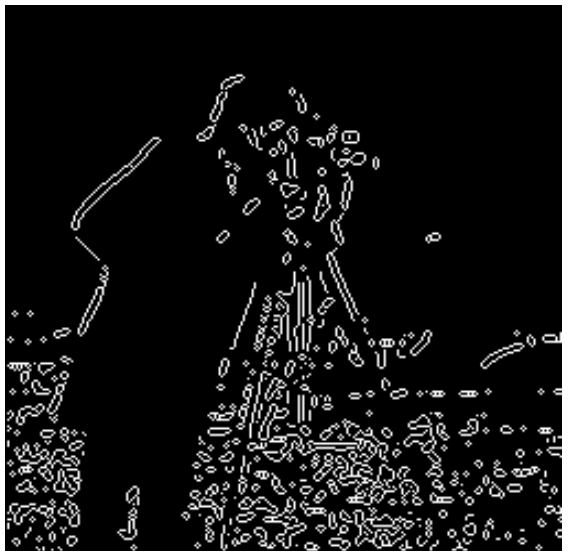

Fig14: Canny

\section{CONCLUSIONS}

Edge detection is one of the most commonly used operations in image analysis. From the comparative study we conclude that the Canny Edge detection performs well compared to Roberts, Prewitt, and Sobel Operators but Canny Detection performance is worse than our propose method known as LookUp Table. Also Canny Detection is more costlier and time consuming. This proposed method known LookUp Table is simple, fast and efficient technique which requires least computational time and gives better results compared to conventional Edge Detection Techniques.

\section{REFERENCES}

[1] M.B. Ahmad and T.S.Choi, Local Threshold and Boolean Function Based Edge Detection, IEEE Transactions on Consumer Electronics, Vol.45, No 3.August 1999.

[2] J.Canny. A computational approach to edge detection, IEEE Transactions in pattern analysis and machine intelligence vol. 8 pp. 679-698, 1986
[3] Chunxi Ma, et.al. ; "An improved Sobel algorithm based filter", Institute of Electrical and Electronics Engineers, 2nd International IEEE conference, China, volume:1, pp,88-93, August 1, 2010.

[4] Dao Qiang Zhanga and Song Can Chena, "A novel kernelized fuzzy C-means algorithm with application in medical image segmentation", Artificial Intelligence in Medicine, vol. 32, 2004, pp.37-50

[5] Dinesh K. Sharma, Loveleen Gaur and Daniel Okunbor,"Image Compression and Feature Extraction with Neural Network", Proceedings of the Academy of Information and Management Sciences, Vol.11, No.1, 2007, pp. 33-38.

[6] R.C.Gonzalez and R.E.Woods, Digital Image Processing 2nd ed. Prentice Hall, 2002. Jianbo Shi; Jitendra Malik, "Normalized Cuts and Image Segmentation",IEEE Trans. Pattern Analysis and Machine Intelligence. Vol 22, No 8, 2000

[7] M.Ibrahiem, M.EL Emery, On the application of Artificial Neural in analysing and classifying human chromosome, Journal of Computer science vol. 2(1) pp. 72-75 2006.

[8] R.Maini, H.Aggarwal. Study and comparison of various image edge detection techniques, International Journal of Image processing (IJIP), volume (3)

[9] Mantas Paulinas and Andrius Usinskas, "A Survey of Genetic Algorithms Applicatons for Image Enhancement and Segmentation", Information Technology and Control, vol.36, No.3, 2007, pp.278-284.

[10] D.Marr, E.C.Hildreth. Theory of edge detection, proceeding of the Royal Society, 201b, pp187-217, 1980

[11] S. Price, "Edges: The Canny Edge Detector", July 4, 1996.

[12] A. Rosenfel, Computer vision, a source of models for biological visual process, IEEE Transaction on Biomedical 36(1), pp. 83-94, 1989.

[13] Sobel, Neighbourhood coding of binary images fast contour following and general array binary processing, Computer graphics and image processing vol. 8, pp. 127- 135, 1978.

[14] N. Senthilkumaran and R.Rajesh, A study on edge detection methods for image segmentation Proceedings of the international Conference on Mathematics and Computer Science (ICMCS-2009) vol. 1, pp. 255-259, 2009.

[15] N. Senthilkumaran and R.Rajesh, Edge Detection Techniques for image segmentation-A survey, Proceedings of the international conference on managing next generation software applications (MNGSA-08) pp. 749-760, 2008.

[16] WenshuoGao, et.al. ; "An improved Sobel edge detection", computer science and information technology (ICCSIT), 2010 3rd IEEE International Conference, China, volume: 5, pp. 67-71, 9-11 July 2010.

[17]A. Yuille and T.A.Poggio. Scaling theorems for zero crossing IEEE Transaction on Pattern Anal Machine Intelligence vol. 8, no.1 pp. 157-163, 1986 


\section{BIOGRAPHIES}

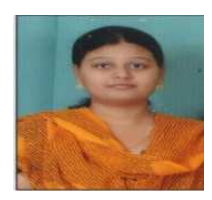

M. Sushma Sri Pursuing M.Tech(WMC) from Jaya Prakash Narayana College of Engineering B.Tech(ECE) from Jaya Prakash Narayana College of Engineering Currently she is working as Lecturer in Govt. Polytechnic College and has 2 years of Experience in teaching. Her areas of interest include, Image processing, Wireless Networks, Signal Processing, Communication.

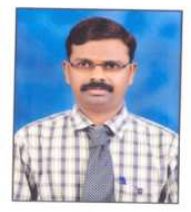

Dr. M. Narayana is a professor and HOD in ECE. He received B.Tech from G. Pullareddy College of Engg, Kurnool, SKU, Ananthapur, and M.Tech. From JNTUH, Hyderabad, AP, India and received Ph.D from JNTUA, Ananthapur, AP. He has fifteen years of experience in teaching undergraduate and post graduate students, and guided more than 20 undergraduate and more than 10 postgraduate thesis. He has produced 8 papers in International Journals, 4 papers in International Conferences and 1 paper in National Conferences. His research interests are in areas of signal and image processing, segmentation, pattern recognition, content based image retrieval. He acted as Resource Person for Workshop/ Conferences. 